\title{
Evidence for weak ferromagnetic moment within the basal plane of hematite natural crystals at low temperature
}

\author{
Fatima Martin-Hernandez \\ Department of Geophysics, Faculty of Physics, Universidad Complutense, 28040, Madrid, Spain \\ Instituto de Geociencias (UCM, CSIC), Faculty of CC. Físicas, Universidad Complutense, Madrid, Spain \\ (fatima@fis.ucm.es)
}

\author{
Ann M. Hirt \\ Institute of Geophysics, ETH-Zurich, Zurich, Switzerland
}

[1] Low-temperature magnetization of hematite within the basal plane has been studied in a collection of natural crystals by means of torque magnetometry. Comparison between the torque curves at room temperature and at $77 \mathrm{~K}$ allows identification of a weak ferromagnetic moment constrained within the basal plane at temperatures well below the Morin transition. Annealing the samples produces the expected reduction of the weak ferromagnetic moment, but there is also a relationship between the ferromagnetic moment before and after annealing. Low-temperature measurements after the annealing experiment reveal the presence of a weak ferromagnetic moment that survives the annealing. This observation suggests the magnetic structure of natural hematite crystals below the Morin transition can still be a carrier of magnetization.

Components : 9,155 words, 8 figures, 4 tables.

Keywords: hematite; low-temperature properties; ferromagnetic moment; anisotropy; magnetic transition; torque magnetometry.

Index Terms: 1518 Magnetic fabrics and anisotropy: Geomagnetism and Paleomagnetism; 1519 Magnetic mineralogy and petrology: Geomagnetism and Paleomagnetism; 1540 Rock and mineral magnetism: Geomagnetism and Paleomagnetism.

Received 22 October 2012; Revised 25 July 2013; Accepted 5 August 2013; Published 4 October 2013.

Martin-Hernandez, F., and A. M. Hirt (2013), Evidence for weak ferromagnetic moment within the basal plane of hematite natural crystals at low temperature, Geochem. Geophys. Geosyst., 14, 4444-4457, doi:10.1002/ggge.20245.

\section{Introduction}

[2] Hematite is an iron oxide $\left(\alpha-\mathrm{Fe}_{2} \mathrm{O}_{3}\right)$ that represents the most oxidized state in the wüstitemagnetite-hematite system. It is present in a multitude of natural environments on Earth and it has also been reported on Mars [e.g., Moore, 2004; Squyres et al., 2009]. Hematite has long been rec- ognized as a stable and reliable carrier of natural remanent magnetization in rocks, which reflects the Earth's magnetic field [e.g., Butler, 1992]. However, in the particular case of sedimentary rocks, it has been postulated that the remanent magnetization carried by hematite falsely records the inclination of the ancient geomagnetic field in an effect that has been named "inclination 
shallowing" or "flattening." Inclination shallowing has important implications for the determination of paleolatitudes, because this will lead to a false paleolatitude, hence geographic position [e.g., Bilardello and Kodama, 2010a; Garces et al., 1996]. Two main methods have been proposed for the correction of inclination. One is based on models of the past geomagnetic field [Kent and Tauxe, 2005; Tauxe et al., 2008] and the second is based on measurements of the magnetic anisotropy [e.g., Bilardello and Kodama, 2010b; Jackson et al., 1991; Kodama and Sun, 1992]. The use of one method or the other depends on technical aspects, such as number of data points, intensity, and quality of the signal or rock magnetic properties. To better understand the role of inclination flattening, better knowledge of the magnetic anisotropy of hematite single crystal is needed.

[3] Hematite is antiferromagnetic (AFM) at room temperature with a small canted moment lying within the crystal symmetry plane or basal plane (weak ferromagnetism, WFM) [e.g., Dunlop and Ozdemir, 1997; Néel, 1953]. At low temperature, hematite undergoes a magnetic phase transition from WFM to a pure antiferromagnetic configuration (AF), which is known as the Morin transition [Morin, 1950]. It has been the object of a multitude of studies since the seminal work by Morin, with transition temperatures ranging from 235 to 263 K (see Morrish [1994] for a comprehensive summary on values and techniques).

[4] A number of earlier studies have reported a remanent magnetization below the Morin transition [Flanders and Scheuele, 1964; Néel, 1953; Tasaki and Iida, 1963a; Tasaki et al., 1960], thus inferring a nonparallel structure of the spins along the $c$ axis. More recently, a magnetoelastic origin of the spins' misalignment has been proposed to explain the observed magnetization [Ozdemir and Dunlop, 2005, 2006]. However, the exact orientation of this weak-ferromagnetic moment at low temperature (WFM-LT), with respect to the room temperature orientation and its relationship to the crystallographic $c$ axes is still unknown.

[5] The behavior of hematite remanence at temperatures below the Morin transition may be important in planetary environments as a possible carrier of magnetization where its presence has been confirmed [Squyres et al., 2009]. Mars is one example in our solar system, in which daily temperature ranges from $27^{\circ} \mathrm{C}(300 \mathrm{~K})$ to $-143^{\circ} \mathrm{C}$ (130 K) [Liu et al., 2003], thus crossing the Morin transition on a daily basis [Morin, 1950]. There are currently two hypotheses that could explain the magnetic anomalies measured in Martian crust. The first proposes three magnetic minerals common also in the Earth's crust (magnetite, hematite, and pyrrhotite) that would carry the remanent magnetization [Kletetschka et al., 2000, 2003]. The second hypothesis suggests that lamellar magnetism, the strong magnetic coupling in hemoilmenite exolutions [Hargraves, 1959], could be the origin of the Martian crustal anomalies [Fabian et al., 2011; Robinson et al., 2002]. The confirmation of a WFM-LT would add another possible source magnetic anomalies in the Martian crust.

[6] This work reports on a set of torque measurements from nine hematite natural crystals at room temperature and at $77 \mathrm{~K}$. The measurements at room temperature are modeled by the theoretical function of torque curves in hematite plates due to the WFM constrained within the basal plane [Martin-Hernandez and Hirt, 2004; Porath and Chamalaun, 1966]. The measurements at $77 \mathrm{~K}$, below the Morin transition, can be divided in two types: (i) crystals that show pure antiferromagnetic behavior, i.e., similar to the torque curves of paramagnetic minerals [Martin-Hernandez and Hirt, 2001] and (ii) crystals that show WFM-LT constrained within the basal plane. Six of the samples were subsequently annealed during $24 \mathrm{~h}$ in order to release accumulated stress, reduce dislocation density, and reduce the defect moment [Dunlop, 1971]. Torque was remeasured after annealing at room temperature for all six crystals and at low temperature for two crystals.

\section{Hematite Magnetic Structure}

[7] Hematite crystallizes in the rhombohedral system, although it is commonly indexed using hexagonal axes, with a triaxial structure in the basal plane (0001) and $c$ axes normal to it [Coey, 2010; Giocavazzo, 2011]. Dzyaloshinsky [1958] explained the presence of a WFM at room temperature using a thermodynamic model based on Landau's theory of phase transition of the second kind [Landau, 1936]. The theory, which was further improved by Moriya [1960] by including the antisymmetric interactions of spin-orbit coupling, predicts three solutions that minimize the thermodynamic potential named States I, II, and III (the latter has only been found empirically in one study [Flanders, 1972]).

[8] For State I, solution of the thermodynamic potential at temperatures below $250 \mathrm{~K}$, the spins are aligned antiferromagnetically parallel to the $c$ 
Table 1. List of Samples, Their Place of Origin, and Experiments Performed on the Crystal ${ }^{\mathrm{a}}$

\begin{tabular}{lll}
\hline Sample & \multicolumn{1}{c}{ Locality } & \multicolumn{1}{c}{ Experiments } \\
\hline EH02a & Elba, Italy & TM high, TM low, HYS, IRM, FORC, HF-AMS RT/LT, AN HF-AMS RT/LT \\
EH03c & Elba, Italy & HYS, IRM, FORC, HF-AMS RT/LT, AN HF-AMS RT/LT \\
GB2b & Gorpibach, Switzerland & TM high, TM low, HYS, IRM, FORC, HF-AMS RT, AN HF-AMS RT \\
GB3 & Gorpibach, Switzerland & TM low, HYS, IRM, FORC, HF-AMS RT/LT \\
GB4 & Gorpibach, Switzerland & TM low, HF-AMS RT/LT, AN HF-AMS RT \\
KUKU & Kuckucksberg, Harghita, Romania & HYS, IRM, FORC, HF-AMS RT/LT, AN HF-AMS RT/LT \\
HEM2a & Elba, Italy & TM high, HYS, IRM, FORC, HF-AMS RT/LT \\
HEM3038 & Madeira, Portugal & HYS, IRM, FORC, HF-AMS RT/LT \\
HEM3044b & Madeira, Portugal & TM high, HYS, IRM, FORC, HF-AMS RT/LT \\
\hline
\end{tabular}


high-field torque; AN: annealed.

axis. It represents the highest symmetry configuration case. The theory, however, does not predict a remanent moment in ideal single crystals.

[9] For Stage II, the spins lie antiferromagnetically within the basal plane at temperatures above 250 $\mathrm{K}$ with a canted moment in the symmetry plane. For Stage III the spins also lie antiferromagnetically within the basal plane, but the canted moment falls within the $a-c$ plane. State II would imply a remanent moment constrained within the basal plane, whereas State III would imply a remanent moment with a projection along the $c$ axis.

[10] In addition to the canted moment at room temperature, hematite exhibits an additional weak, more variable magnetic moment referred as "defect moment" [Dunlop, 1971]. Most of the studies that have focused their attention on the hematite defect moment are based on remanence measurements [Dunlop, 1971; Ozdemir and Dunlop, 2006; Rochette et al., 2005], but also on H-M magnetization curves [Besser et al., 1967] or neutron diffraction [Curry et al., 1965].

\section{Samples and Methods}

[11] Nine natural hematite crystals have been analyzed in this study, and the origin of the samples can be found in Table 1. The magnetic properties of the crystals were analyzed using a combination of the following rock magnetic methods (Table 1): (i) thermomagnetic curves between 77 and $973 \mathrm{~K}$, (ii) acquisition of isothermal remanent magnetization (IRM) and subsequent back-field IRM, (iii) hysteresis loops up to $1 \mathrm{~T}$, and (iv) first-order reversal curve (FORC) analysis within the basal plane of the crystal. Thermomagnetic curves at high temperature were carried out with a Vibrating Field Translation Balance (VFTB) [Krasa et al., 2007] for samples HEM 2 and HM3044b. IRM acquisition curves, back-field IRM, hysteresis loops, and FORC diagrams were measured on a MicroMag 3900 Vibrating Sample Magnetometer (VSM) manufactured by Princeton Measurements Corporation. Low-field magnetic susceptibility was measured at low temperatures on an AGICO KLY2 susceptibility bridge for samples GB3, HEM2, KUKU, and EH03A in order to determine the antiferromagnetic nature of the crystals at temperatures below the Morin transition.

\subsection{High-Field Torque Magnetometer}

[12] Samples were measured on a high-field torque magnetometer in at least five applied fields with angular steps of $20^{\circ}$ or $30^{\circ}$ at the Laboratory of Natural Magnetism (LNM), ETH-Zurich, Switzerland [Bergmüller et al., 1994]. The measurement requires the sample to be measured in three mutually perpendicular positions in which one plane is the basal plane of the crystal. The samples were first measured at room temperature and subsequently measured at $77 \mathrm{~K}$ following the measurements protocols described by Martin-Hernandez and Hirt [2001] and Schmidt et al. [2007b], respectively.

[13] There is little literature about high-field torque magnetometry in the field of rock magnetism, but a good technical summary can be found in works such as Bhathal [1971] and Collinson [1983]. The magnetic torque determined in hematite and hematite-bearing rocks has been nicely summarized by Bhathal [1971] and Stacey and Banerjee [1974].

[14] When a magnetic field (B) is applied, the torque (t) can be computed as

$$
\mathbf{t}=\mathbf{m} \times \mathbf{B}
$$

where $\mathbf{m}$ is the magnetization of the sample with an applied field and $\mathbf{B}$ is the applied field. Paramagnetic and diamagnetic minerals have a 
magnetization that is linearly dependent with an applied field, with a proportionality constant defined by the susceptibility tensor:

$$
m_{i}=k_{i j} H_{j} \forall i, j=1,2,3
$$

$m_{i}$ is the magnetization along the $i$ direction, $k_{i j}$ is the $(i, j)$ term of the symmetric susceptibility tensor, and $H_{j}$ is the applied field along the $j$ direction. Applying equation (2), the magnetic torque will be proportional to the square of the applied field [Martin-Hernandez and Hirt, 2001; Owens and Bamford, 1976]. A sample rotated in the $x_{1}-x_{2}$ plane, where the magnetic field is applied, would experience a torque in the perpendicular direction given by

$$
t_{3}(\theta, B)=\frac{1}{2 \mu_{o}} B^{2}\left[\left(k_{22}-k_{11}\right) \sin 2 \theta+2 k_{12} \cos 2 \theta\right]
$$

where $t_{3}$ is the magnetic torque along the $x_{3}$ direction, $B$ is the applied field, $k_{i j}$ are the terms of the paramagnetic susceptibility tensor, and $\theta$ is the angle between the applied field and the $x_{1}$ direction. The torque signal is characterized by a periodic curve (Figure 1a) with increasing amplitude as a function of increasing the applied field. The amplitude can be fitted using a second-order polynomial with only the quadratic term (Figure 1b).

[15] Ferri/ferromagnetic minerals above their saturation have a magnetization, which is constant with applied field. Magnetic anisotropy is a function of differences in the demagnetization factor as well as the torque signal, which is constant with increasing applied fields [Martin-Hernandez and Hirt, 2001; Owens and Bamford, 1976]. Similar to the previous example, a sample rotated in the $x_{1}-x_{2}$ plane, in which the magnetic field is applied, would experience a torque in the perpendicular direction given by

$$
t_{3}=\frac{1}{2 \mu_{o}} M_{s}^{2}\left[\left(N_{22}-N_{11}\right) \sin 2 \theta+2 N_{12} \cos 2 \theta\right]
$$

where $t_{3}$ is the magnetic torque, $M_{s}$ is the saturation magnetization of the ferri/ferromagnetic phase, and $N_{i j}$ is the $(i, j)$ term of the demagnetization tensor. The torque curve is characterized by a periodic curve (Figure 1c), which does not increase its amplitude as the strength of the applied field is increased (Figure 1d).

[16] The magnetization in hematite crystals at room temperature is expressed as the sum of two terms
[Lin, 1959a; Tasaki and Iida, 1963b; Townsend, 1920]:

$$
m=m_{o}+\chi H
$$

where $\chi$ is the magnetic susceptibility and $H$ is the applied field. The first term $m_{\mathrm{o}}$ is the weak ferromagnetic moment constrained within the basal plane that arises from a canting effect $\left(m_{\mathrm{o}}\right)$, and the second term is the induced magnetization due to the applied field. The torque of a sample at a certain applied field can be described by a firstorder term that depends on the applied field and the weak ferromagnetic moment, and a secondorder term that depends on the square of the applied field and the magnetic susceptibility [Kaczer and Shalnikova, 1964; Martin-Hernandez and Hirt, 2004; Porath and Chamalaun, 1966].

[17] The torque attributed to the weak ferromagnetic moment in a plane different than the basal plane is described by

$$
t(\theta)=\operatorname{sign}\left[\cos \left(\theta-\theta_{o}\right)\right] \frac{m_{o} B \sin \left(\theta-\theta_{o}\right) \sin ^{2} \varphi}{\sqrt{1+\tan ^{2}\left(\theta-\theta_{o}\right) \cos ^{2} \varphi}}
$$

where $\theta$ is the orientation of the hematite with respect to the sample coordinates, $\theta_{\mathrm{o}}$ is the initial orientation of the weak ferromagnetism with respect to the sample coordinates, $m_{\mathrm{o}}$ is the constant term of the WFM, $\varphi$ is the angle between the hematite basal plane and the plane of rotation, and $B$ is the applied field (Figure 2). Torque curves for hematite crystal and hematite bearing rocks are characterized by a drop in the torque signal when the term $\operatorname{sign}\left[\cos \left(\theta-\theta_{o}\right)\right]$ changes value with torque drastically changing being +1 when the argument $\cos \left(\theta-\theta_{\mathrm{o}}\right)$ is positive and vice versa.

[18] The torque from an antiferromagnetic material depends on the square of the applied field and its generic torque can be described by

$$
t(\theta)=\frac{1}{2 \mu_{o}} B^{2}\left[\left(\chi_{\perp}-\chi_{\|}\right) \sin 2 \theta\right]
$$

where $\chi_{\perp}$ is the magnetic susceptibility perpendicular to the easy axes and $\chi_{\|}$is the magnetic susceptibility parallel to the easy axes within the plane of measurement.

[19] The torque curve is characterized by a periodic function with a $2 \theta$ component. Figure $1 \mathrm{e}$ shows a typical torque curve for a hematite single crystal (or hematite-bearing rock) featuring a 

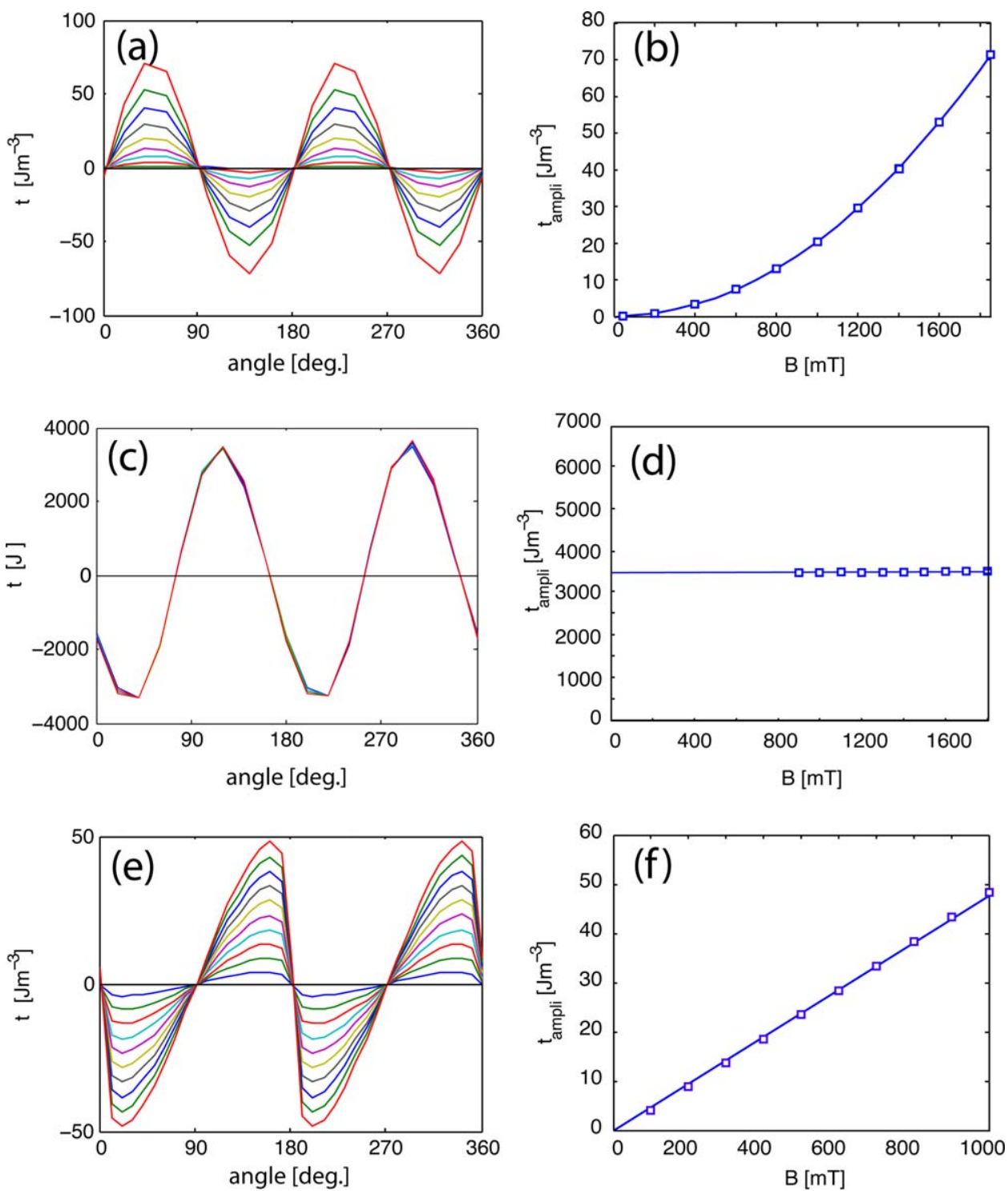

Figure 1. (a, c, and e) Torque as a function of increasing applied field and angle and (b, d, and f) corresponding amplitude of the torque signal as a function of applied field for three typical magnetic behavior examples. Biotite single crystal with typical paramagnetic behavior (Figures 1a and 1b), hornblende crystal with a strong concentration of ferromagnetic inclusions above their saturation (Figures 1c and 1d) and hematite single crystal with typical canted antiferromagnetic behavior (Figures 1e and 1f).

sudden drop in the magnetization after the maximum torque value that coincides with a $180^{\circ}$ change in the direction of the WFM within the basal plane [Porath and Chamalaun, 1966]. The amplitude of the torque signal increases linearly with increasing applied fields as predicted by the proposed model (Figure 1f).

[20] The torque amplitude at room temperature for every measurement angle has been fitted as a function of field strength into a polynomial of second order without ordinate. The fitting is automatic unless the correlation coefficient is lower than 0.97 ; N.B. the fitting was always automatic in this study. The linear coefficient has been fitted as a function of angular position using equation (6). The orientation of the magnetization and its magnitude have been computed using a least squares nonlinear fitting of the curve with a MATLAB® subroutine developed by Martin-Hernandez and Hirt [2004].

[21] The torque curve at different applied fields has also been measured below the Morin transition at $77 \mathrm{~K}$ [Schmidt et al., 2007a, 2007b]. Similarly, the torque amplitude at all measuring angles has been fitted as a polynomial as a function of applied field. Some samples possessed a significant linear 


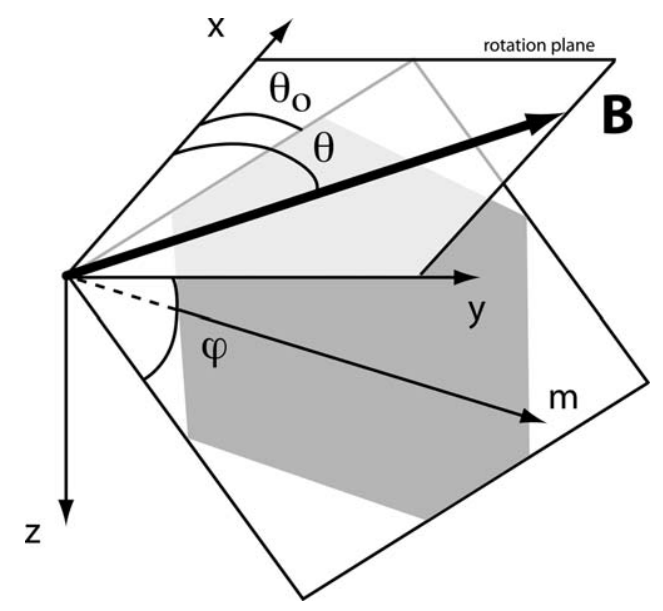

Figure 2. Schematic diagram of the angular relationship between the hematite crystal, the orientation of the weak ferromagnetic moment within it $(\mathrm{m})$, the plane of measurement $(x-y)$, and the sample coordinate system. When $\mathbf{B}$ is the applied field, $\theta$ is the angle between the field $\mathbf{B}$ and the $x$ direction of the sample coordinate system, $\theta_{0}$ is the angle between the measuring plane $x-y$ and the intersection of the crystal and this plane, and $\varphi$ is the angle between the rotation plane and the crystal basal plane.

term of the polynomial, which has been fitted into the theoretical torque curve attributed to hematite in a WFM state (equation (6)). Other samples showed only a torque amplitude dependence on the square of the applied field, related to the magnetic susceptibility of hematite in an AF state [Martin-Hernandez and Hirt, 2001]. Torque curves in samples showing this behavior could be fitted using a series of trigonometric functions derived using Fourier analysis (equation (7)).

[22] Six of the samples were annealed in a Schoensted oven for $24 \mathrm{~h}$ at $650^{\circ} \mathrm{C}$. After cooling the samples in a zero-field environment, the highfield anisotropy was measured at room temperature for all samples and at $77 \mathrm{~K}$ for three samples. The mathematical treatment of the signal is the same as described above.

\section{Results}

\subsection{Rock Magnetism}

[23] Thermomagnetic curves allow the determination of the Néel temperature of hematite. A small chip from samples GB2b, HEM2, and HM3044b was measured up to $700^{\circ} \mathrm{C}$ in order to define the temperature at which magnetization is lost. The three samples showed a Néel temperature of $680^{\circ} \mathrm{C}$ (Figure 3a) compatible with pure hematite [Dunlop and $\ddot{O} z d e m i r, 1997]$. Low-temperature thermomag- netic curves were run on four samples and showed a Morin transition between 240 and $250 \mathrm{~K}$. For example, samples GB3 and HEM2 (Figures 4a and 4b) show a drop in magnetization at $260 \mathrm{~K}$ $\left(-13^{\circ} \mathrm{C}\right)$, characteristic of hematite Morin transition [Morin, 1950]. Samples KUKU and EH02A display a lower temperature Morin transition at about $240 \mathrm{~K}\left(-33^{\circ} \mathrm{C}\right)$ (Figures $4 \mathrm{c}$ and $\left.4 \mathrm{~d}\right)$.

[24] IRM acquisition curves and subsequent DC backfield IRM demagnetization show the same feature in all samples (Figure 3b). Magnetization increases with applied field and saturation in the basal plane is reached at approximately $600 \mathrm{mT}$. The coercivity of remanence is very similar in all samples with values that range from 12.1 to $70.1 \mathrm{mT}$ (Table 2), which is compatible with hematite [Peters and Dekkers, 2003] and in particular with hematite single crystals [Martin-Hernandez and GuerreroSuárez, 2012; Ozdemir and Dunlop, 2006]. FORC diagrams measured within the hematite basal plane are characterized by a low coercivity also compatible with the bulk coercivity, obtained from the hysteresis loops (Figure 3d). The diagrams show two features that have been already reported in hematite single crystals. First, the maximum value of the diagram shifted toward negative values of $H_{\mathrm{u}}$ [MartinHernandez and Guerrero-Suárez, 2012]. Second, the shape of the maximum intensity is asymmetric following a pattern described by Brownlee et al. [2011] as "kidney shaped."

\subsection{Room Temperature Torque Curves}

[25] The nine samples show similar features when torque curves are measured in a plane perpendicular to the basal plane (Figure 5). Here amplitude of the torque signal increases as a function of angle until it reaches a position where a sudden drop in torque occurs (Figure 5a). This coincides with the measurement within the basal plane where magnetization changes direction [Flanders and Remeika, 1965; Kaczer and Shalnikova, 1964; Porath and Chamalaun, 1966; Tasaki et al., 1962]. At any measurement angle, the torque amplitude increases with the applied field (Figure $5 b)$. Torque as a function of field can be fitted by a polynomial of second order with no ordinate. The linear term originates in the WFM of hematite (equation (6)) and the second-order term is attributed the magnetic susceptibility of the antiferromagnet (equation (7)). The linear coefficient of the polynomial fitting, when plotted as a function of angular position, represents the torque per field exerted by the WFM moment constrained in the basal plane (Figure 5c). A least squares, nonlinear 

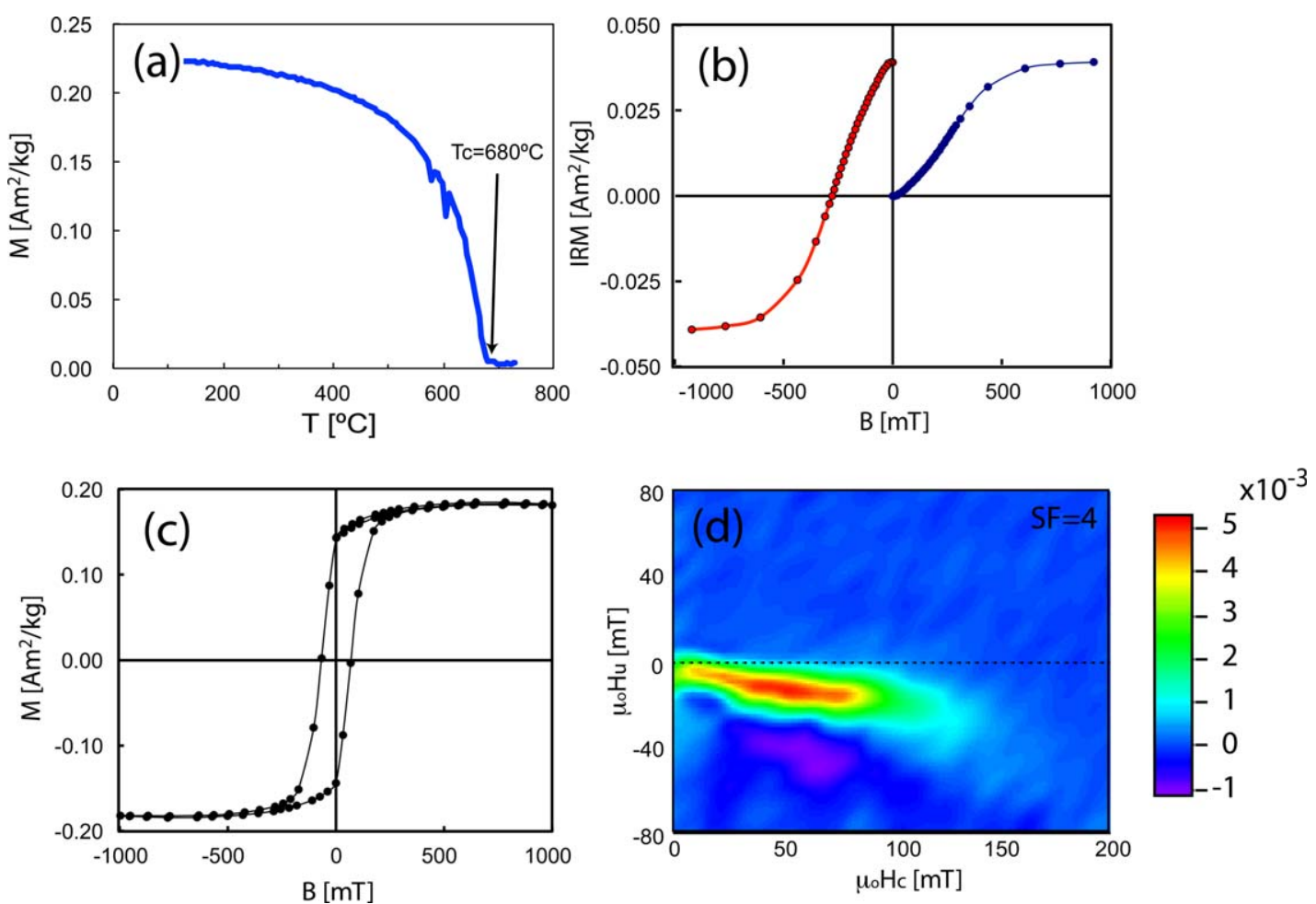

Figure 3. Summary of rock magnetic properties within the basal plane for sample HM3044b. (a) Thermomagnetic curve from room temperature to $700^{\circ} \mathrm{C}$ showing the Curie temperature at $680^{\circ} \mathrm{C}$, (b) IRM acquisition curve and subsequent back-field IRM demagnetization, (c) hysteresis loop after subtraction of the susceptibility of the reversible part, and (d) FORC diagram with an smoothing factor (SF) of 4.
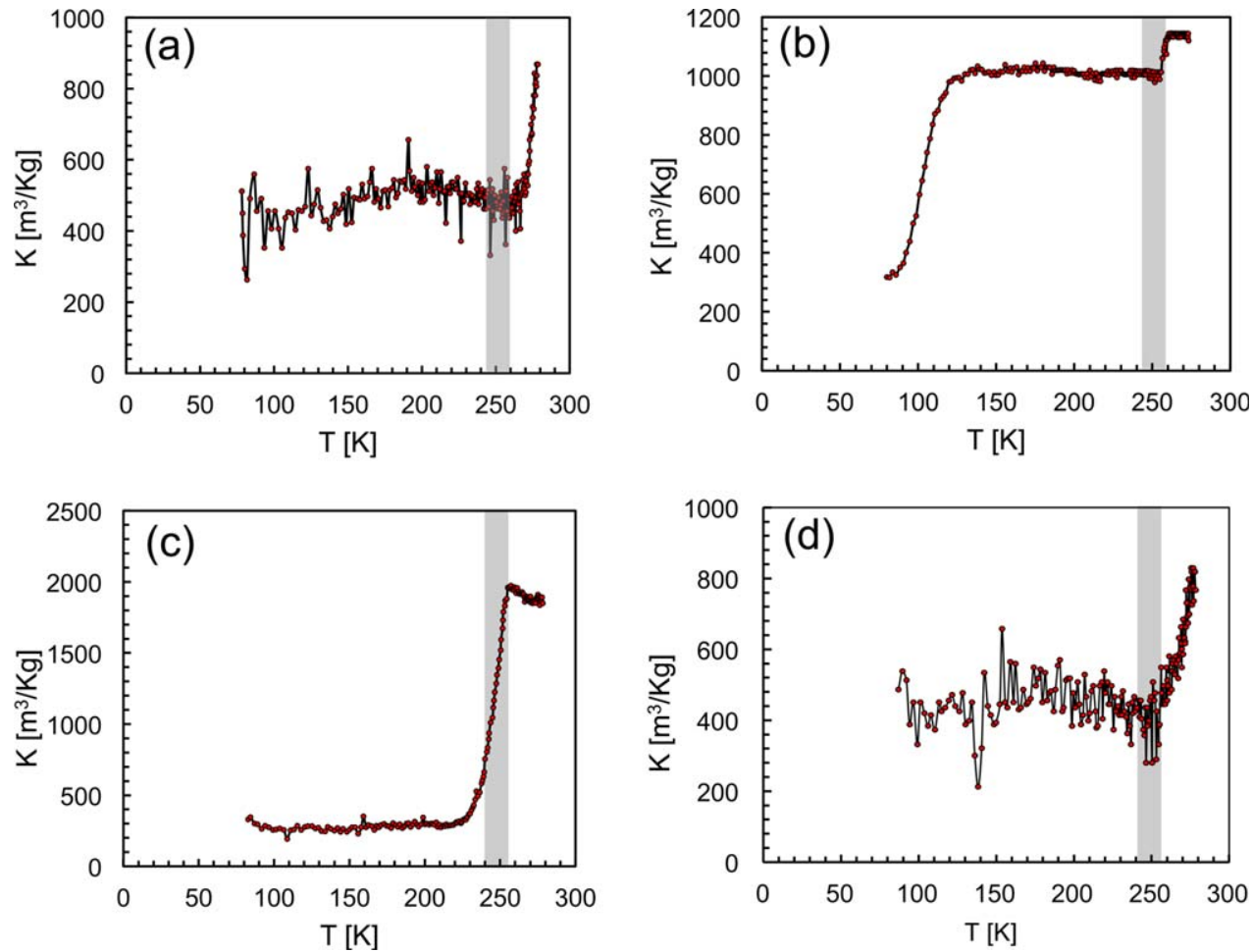

Figure 4. Low-field susceptibility measurements within the basal plane from room temperature to $77 \mathrm{~K}$ for samples (a) GB3, (b) HEM2, (c) KUKU, and (d) EH02A. 
Table 2. Rock Magnetic Properties of the Hematite Single Crystals Derived From Hysteresis Loops ${ }^{\mathrm{a}}$

\begin{tabular}{|c|c|c|c|c|c|c|c|}
\hline Sample & Weight (g) & $M_{\mathrm{r}}\left(\mathrm{Am}^{2} / \mathrm{kg}\right)$ & $M_{\mathrm{s}}\left(\mathrm{Am}^{2} / \mathrm{kg}\right)$ & $H_{\mathrm{cr}}(\mathrm{mT})$ & $H_{\mathrm{c}}(\mathrm{mT})$ & $H_{\mathrm{cr}} / H_{\mathrm{c}}$ & $M_{\mathrm{r}} / M_{\mathrm{s}}$ \\
\hline EH02A & 0.10560 & 0.27225 & 0.70938 & 17.8 & 14.0 & 1.27 & 0.38 \\
\hline EH03C & 0.01400 & 0.15464 & 0.23943 & 69.6 & 55.0 & 1.27 & 0.65 \\
\hline GB2B & 0.16280 & 0.17881 & 0.32432 & 24.3 & 20.3 & 1.20 & 0.55 \\
\hline GB3 & 0.05360 & 0.20075 & 0.27817 & 79.5 & 55.2 & 1.44 & 0.72 \\
\hline GB4 & 0.18290 & $\sim$ & $\sim$ & $\sim$ & $\sim$ & $\sim$ & $\sim$ \\
\hline KUKU & 0.08030 & 0.26772 & 0.32468 & 84.0 & 52.4 & 1.60 & 0.82 \\
\hline HEM2A & 0.15591 & 0.20244 & 0.28844 & 29.0 & 24.9 & 1.16 & 0.70 \\
\hline HM3038 & 0.32310 & 0.04493 & 0.04720 & 12.3 & 10.0 & 1.23 & 0.95 \\
\hline HM3044b & 0.13220 & 0.13900 & 0.18267 & 70.7 & 67.9 & 1.04 & 0.76 \\
\hline
\end{tabular}

${ }^{a} M_{r}$ is the remanent magnetization, $M_{s}$ is the saturation magnetization, $H_{c}$ is the coercivity field, and $H_{c r}$ is coercivity of remanence.

fitting of the data for two perpendicular measurement planes, which are different from the basal plane, into the theoretical curve provides information about the magnitude and initial orientation of the ferromagnetic moment (Figure $5 \mathrm{c}$ and Table $3)$. The ferromagnetic moment ranges in magnitude from approximately $900 \mathrm{~A} / \mathrm{m}$ to a maximum value of $2923 \mathrm{~A} / \mathrm{m}$, similar to specific measurements of the weak ferromagnetic moment in hematite [Morrish, 1994]. The computed ferromagnetic moment tends to lie in the direction of the first applied field, i.e., the declination is close to zero, the value of the first orientation. Schmidt and Fuller [1970] also noted this when examining magnetic anisotropy in the basal plane of hematite with low field methods. The inclination of the magnetization, with respect to the hematite basal plane is very flat, with a maximum deflection of $17^{\circ}$, which is within the uncertainly of the orientation of hematite crystals (Table 3 ).

\subsection{Low-Temperature Torque Curves}

[26] Eight of the natural crystals have been measured at liquid nitrogen temperature (Figure 6).
Measurements in a plane perpendicular to the basal plane show two different types of behavior. The torque curves as a function of applied field for samples HEM2A and HM3038 have a symmetrical shape (Figure 6a). At any given measurement angle, the torque amplitude depends only on the square of the applied field (Figure 6b). The proportionality coefficient derived from the fitting, when plotted as a function of angular orientation, can be modeled with a symmetric trigonometric function with a frequency of $2 \theta$ (Figure 6c). These samples, therefore, show no WFM-LT below the Morin transition.

[27] The other six samples showed a different torque signal when measured as a function of orientation. The curve is similar to the measurements at room temperature, with a strong asymmetry with respect to the vertical axes (Figure 6d). At one fixed angular position, the torque can be fitted as a function of applied field into a second-order polynomial with a significant linear term (Figure 6e). The computed linear coefficient, when represented as a function of orientation can be fitted using the model of torque for a weak ferromagnetic moment proposed for hematite at room temperature (Figure 6f).


Figure 5. Summary of torque measurements at room temperature in a plane perpendicular to the hematite basal plane at different applied fields for sample HM3044b. (a) Torque curve of a hematite crystal within the basal plane as a function of applied field for fields ranging from 0.6 to $1.5 \mathrm{~T}$ at $100 \mathrm{mT}$ step, (b) amplitude of the torque signal as a function of applied field at one fixed angle of $30^{\circ}$ with respect to the origin and least square polynomial fitting, and (c) linear component of the torque signal as a function of the applied field and fitting into the theoretical function described by Martin-Hernandez and Hirt [2004]. 
Table 3. Ferromagnetic Moment Constrained Within the Basal Plane of Hematite Natural Crystals Derived From Torque Curves and Initial Orientation (Declination D and Inclination I) of the Weak Ferromagnetic Moment Within the Basal Plane in the Sample Coordinate System ${ }^{\mathrm{a}}$

\begin{tabular}{|c|c|c|c|c|c|c|c|c|c|c|c|c|c|}
\hline \multirow[b]{2}{*}{ Sample } & \multirow[b]{2}{*}{ Mass (gr) } & \multicolumn{6}{|c|}{$300 \mathrm{~K}$} & \multicolumn{6}{|c|}{$77 \mathrm{~K}$} \\
\hline & & $\begin{array}{c}m_{1} \\
(\mathrm{~A} / \mathrm{m})\end{array}$ & $\begin{array}{c}I_{1} \\
(\operatorname{deg})\end{array}$ & $\begin{array}{c}D_{1} \\
(\operatorname{deg})\end{array}$ & $\begin{array}{c}m_{2} \\
(\mathrm{~A} / \mathrm{m})\end{array}$ & $\begin{array}{c}I_{2} \\
(\mathrm{deg})\end{array}$ & $\begin{array}{c}D_{2} \\
(\operatorname{deg})\end{array}$ & $\begin{array}{c}m_{1} \\
(\mathrm{~A} / \mathrm{m})\end{array}$ & $\begin{array}{c}I_{1} \\
(\operatorname{deg})\end{array}$ & $\begin{array}{c}D_{1} \\
(\operatorname{deg})\end{array}$ & $\begin{array}{c}m_{2} \\
(\mathrm{~A} / \mathrm{m})\end{array}$ & $\begin{array}{c}I_{2} \\
(\mathrm{deg})\end{array}$ & $\begin{array}{c}D_{2} \\
\text { (deg) }\end{array}$ \\
\hline EH02A & 0.1056 & 2147 & 16.3 & 1.5 & 2382 & 5.9 & 5.5 & 1766 & 43.0 & 12.9 & $x$ & $x$ & $\times$ \\
\hline $\mathrm{EH} 03 \mathrm{C}$ & 0.0140 & 869 & 0.0 & 0.0 & 1007 & 11.5 & 15.0 & $x$ & $x$ & $x$ & $x$ & $x$ & $x$ \\
\hline GB2B & 0.0952 & 2923 & 4.0 & 4.1 & 2650 & 4.9 & 2.5 & $\sim$ & $\sim$ & $\sim$ & $\sim$ & $\sim$ & $\sim$ \\
\hline GB3 & 0.2889 & 2916 & 13.6 & 1.9 & 2851 & 11.1 & 1.0 & 2393 & 0.0 & 0.0 & 2196 & 0.0 & 0.0 \\
\hline GB4 & 0.2960 & 2368 & 17.0 & 2.4 & 2412 & 17.9 & 1.9 & 1070 & 19.8 & 7.0 & 1018 & 19.7 & 5.9 \\
\hline KUKU & 0.0432 & 2237 & 10.1 & 2.7 & 2097 & 0.0 & 4.2 & 1812 & 0.1 & 0.0 & 1859 & 0.1 & 0.1 \\
\hline HEM2A & 0.1559 & 1000 & 7.0 & 12.1 & 1021 & 8.3 & 12.3 & \multicolumn{6}{|c|}{ No ferromagnetic moment } \\
\hline HM3038 & 0.3231 & 1787 & 0.1 & 0.1 & 1833 & 4.1 & 2.5 & & & erromas & tic mon & & \\
\hline HM3044b & 0.1322 & 1258 & 6.7 & 5.1 & 1433 & 9.0 & 9.7 & 1291 & 8.9 & 10.0 & 1288 & 11.8 & 10.6 \\
\hline
\end{tabular}

${ }^{a}$ Measurements are done at two temperatures above $(300 \mathrm{~K})$ and below $(77 \mathrm{~K})$ the Morin transition of hematite. Tilde symbol is shown in samples where the measurement was not possible for technical reasons and cross symbol measurements where the proposed model for the magnetization did not converge.

[28] Table 3 summarizes the magnitude and initial orientation of the WFM of samples, where a WFM moment has been modeled at temperatures below the Morin transition. Samples EH02A and EH03C had a very noisy signal when measured at low temperature. The magnitude of the ferromagnetic
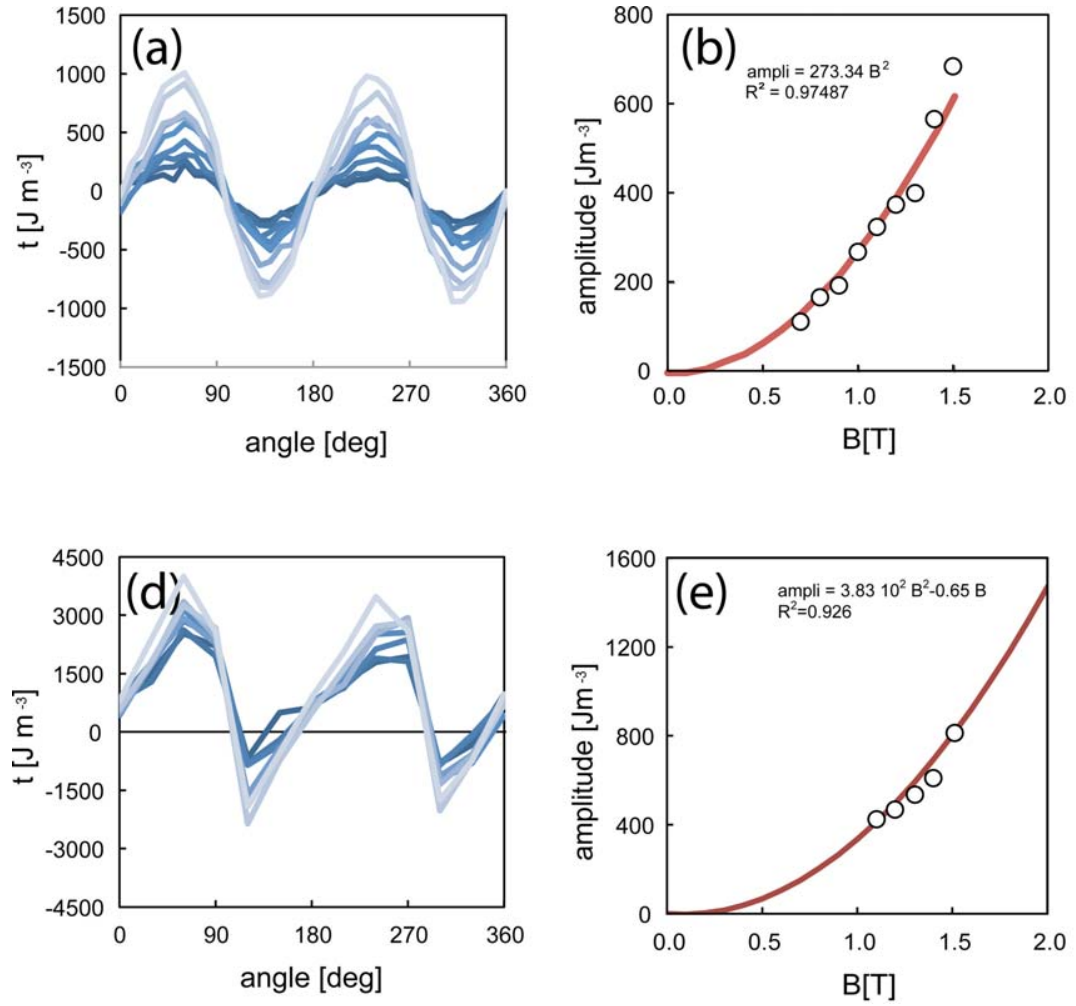
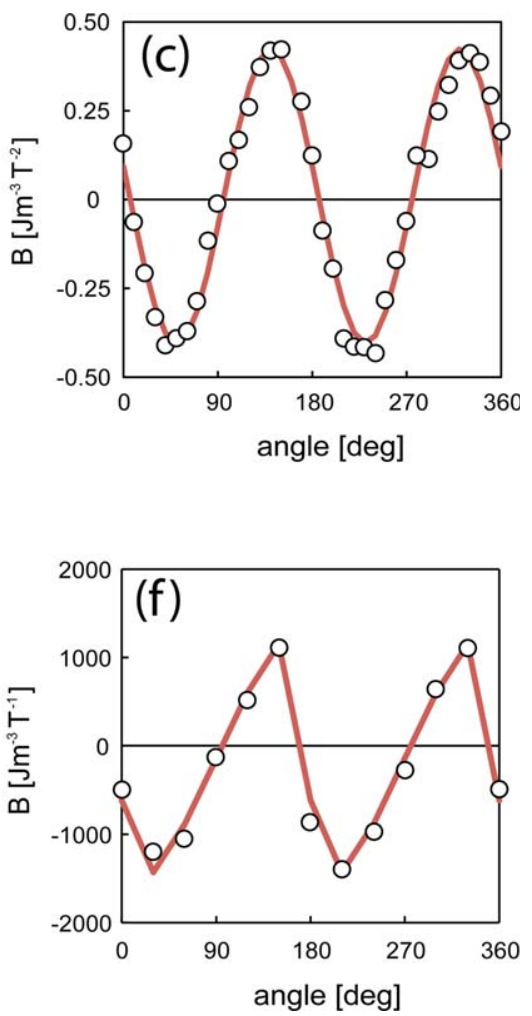

Figure 6. Comparison between the torque curve at $77 \mathrm{~K}$ for $(\mathrm{a}-\mathrm{c})$ sample HEM 2 with no indication of weak ferromagnetic moment and $(\mathrm{d}-\mathrm{f})$ sample KUKU with a strong indication of weak ferromagnetic moment within the basal plane measured in a plane perpendicular to the hematite basal plane. Figures $6 \mathrm{a}$ and $6 \mathrm{~d}$ show the torque curve as a function of the applied field at low temperature, Figures $6 \mathrm{~b}$ and $6 \mathrm{e}$ show the amplitude of the torque signal at one angular position as a function of applied field and fitting into the best polynomial function. Figures $6 \mathrm{c}$ and $6 \mathrm{f}$ are the main coefficients of the polynomial fitting as a function of angular position and best theoretical fitting, where Figure $6 \mathrm{c}$ has been modeled as a trigonometric function by Fourier analysis and Figure $6 \mathrm{f}$ has been modeled with the model proposed by Martin-Hernandez and Hirt [2004]. 

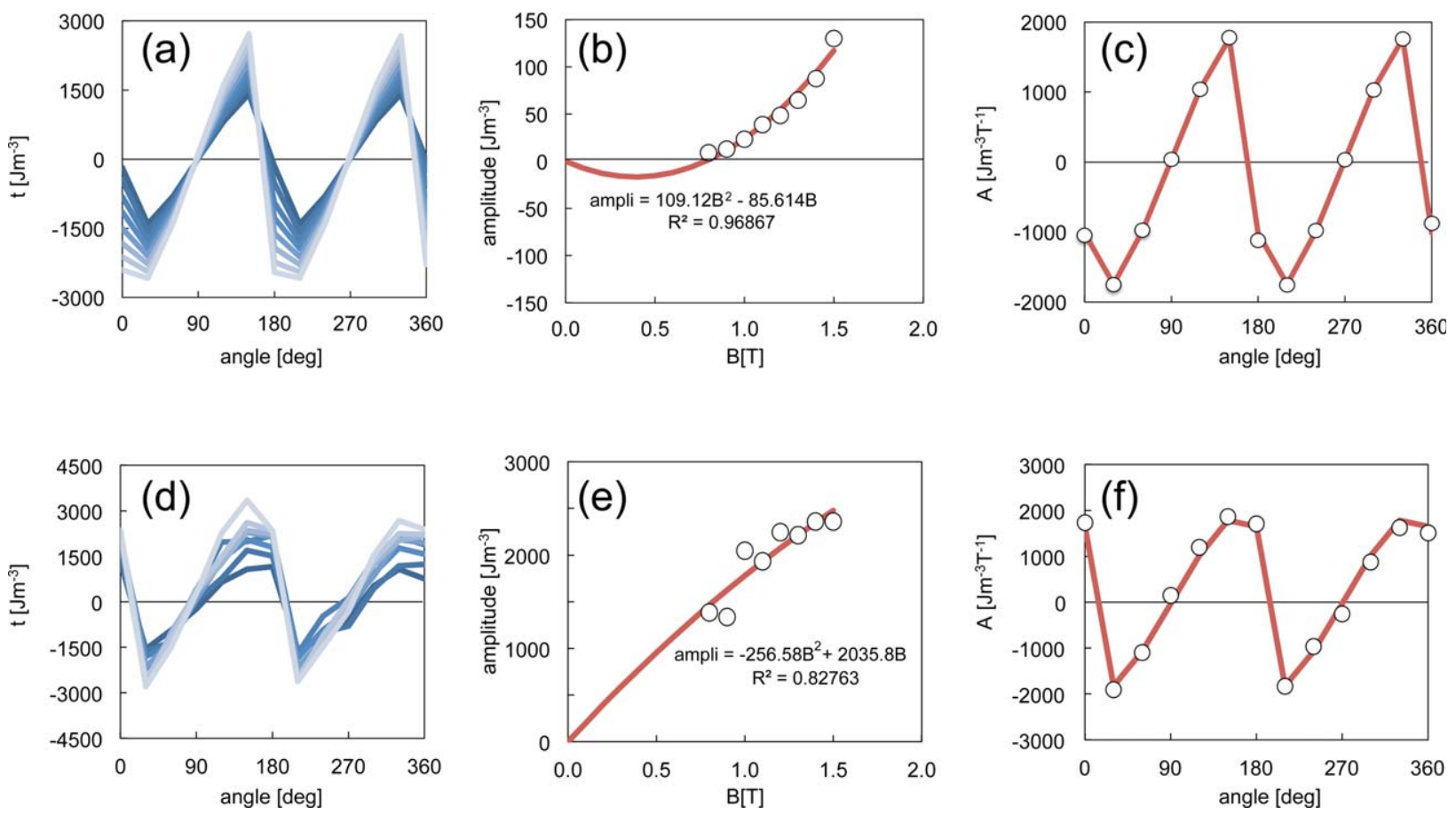

Figure 7. Comparison between the torque curves at $(a-c)$ room temperature and $(d-f)$ low temperature for sample KUKU after annealing at $650^{\circ} \mathrm{C}$ during $24 \mathrm{~h}$.

moment at $77 \mathrm{~K}$ ranges from $1018 \mathrm{~A} / \mathrm{m}$ in sample GB4 to $2393 \mathrm{~A} / \mathrm{m}$ in sample GB3. The moment is always smaller than the magnetization computed at room temperature for all the analyzed samples. The initial orientation of the weak ferromagnetic moment is similar to the direction of the first applied field in the measurement protocol.

\subsection{Annealing Experiments}

[29] Six samples have been annealed for $24 \mathrm{~h}$ in a magnetically shielded environment in order to reduce the intralattice stress, dislocation, and defects. The magnetic moment of hematite is known to decrease after annealing [Dunlop, 1971]. Measurements at room temperature after annealing show similar features to the samples before heating (Figures $7 \mathrm{a}-7 \mathrm{c}$ ). The torque curves are characteristic of hematite crystals and hematite bearing rocks when measured in a plane perpendicular to the basal plane (Figure 7). They are periodic curves characterized by a sudden drop in the torque value when the field is applied in the direction containing the basal plane, similar to those described by Owens [1981] (Figure 7a) and an amplitude of the torque signal that depends on both the square of the applied field and applied field (Figure 7b). The linear coefficient of the torque amplitude when represented as a function of applied field shows the characteristic feature of the torque due to a weak ferromagnetic moment constrained in the crystals basal plane (Figure 7c). It can be satisfactory fitted using the corresponding theoretical function (equation (6)).

[30] Table 4 summarizes the three parameters that describe the weak ferromagnetic moment by means of torque magnetometry. The magnitude of the magnetization after annealing measured at room temperature is lower than measurements before annealing (Tables 3 and 4). However, despite the general decrease of magnetic moment associated with the annealing experiments, the initial direction of the weak ferromagnetic moment remains very similar before and after heating. Annealing decreases the magnetic moment determined by torque magnetometry, with the exception of sample KUKU.

[31] Three of the samples have also been measured at low temperature after being annealed (Figures $7 \mathrm{~d}-7 \mathrm{f}$ and Table 4). Although the torque curve is weaker than the measurements at $77 \mathrm{~K}$ before annealing the samples, they still show the characteristics of the weak ferromagnetism (Figure 7d). The amplitude of the torque signal also depends on both the square of the applied field and the applied field (Figure 7e). The linear term of the polynomial fitting of torque as a function of field 
Table 4. Ferromagnetic Moment Constrained Within the Basal Plane of Hematite and First Anisotropy Constant After Annealing the Samples in a Zero Field Environment for $24 \mathrm{~h}$ at $650^{\circ} \mathrm{C}^{\mathrm{a}}$

\begin{tabular}{|c|c|c|c|c|c|c|c|c|c|c|c|c|c|}
\hline \multirow[b]{2}{*}{ Sample } & \multirow[b]{2}{*}{$\begin{array}{l}\text { Mass After } \\
\text { Anneal (g) }\end{array}$} & \multicolumn{6}{|c|}{$300 \mathrm{~K}$} & \multicolumn{6}{|c|}{$77 \mathrm{~K}$} \\
\hline & & $\begin{array}{l}m_{1}{ }^{\mathrm{a}} \\
(\mathrm{A} / \mathrm{m})\end{array}$ & $\begin{array}{c}I_{1}{ }^{\mathrm{a}} \\
(\mathrm{deg})\end{array}$ & $\begin{array}{l}D_{1}^{\mathrm{a}} \\
(\mathrm{deg})\end{array}$ & $\begin{array}{l}m_{2}{ }^{\mathrm{a}} \\
(\mathrm{A} / \mathrm{m})\end{array}$ & $\begin{array}{l}I_{2}^{\mathrm{a}} \\
(\mathrm{deg})\end{array}$ & $\begin{array}{l}D_{2}{ }^{\mathrm{a}} \\
(\mathrm{deg})\end{array}$ & $\begin{array}{l}m_{1}{ }^{\mathrm{a}} \\
(\mathrm{A} / \mathrm{m})\end{array}$ & $\begin{array}{l}I_{1}^{\mathrm{a}} \\
(\mathrm{deg})\end{array}$ & $\begin{array}{l}D_{1}^{\mathrm{a}} \\
(\mathrm{deg})\end{array}$ & $\begin{array}{l}m_{2}{ }^{\mathrm{a}} \\
(\mathrm{A} / \mathrm{m})\end{array}$ & $\begin{array}{l}I_{2}{ }^{\mathrm{a}} \\
(\mathrm{deg})\end{array}$ & $\begin{array}{l}D_{2}{ }^{a} \\
(\mathrm{deg})\end{array}$ \\
\hline EH02A & 0.1056 & 210 & 16.0 & 1.4 & 198 & 8.1 & 10.7 & 182 & 21.4 & 11.1 & $x$ & $x$ & $x$ \\
\hline EH03C & 0.0140 & 837 & 8.3 & 21.2 & 900 & 18.3 & 4.7 & $x$ & $x$ & $x$ & $x$ & $x$ & $x$ \\
\hline GB2B & 0.0952 & 2482 & 1.9 & 2.0 & 2674 & 15.6 & 0.3 & $\sim$ & $\sim$ & $\sim$ & $\sim$ & $\sim$ & $\sim$ \\
\hline GB3 & 0.2889 & 237 & 13.3 & 13.4 & 290 & 20.6 & 1.8 & $\sim$ & $\sim$ & $\sim$ & $\sim$ & $\sim$ & $\sim$ \\
\hline KUKU & 0.0420 & 2096 & 2.4 & 1.4 & 2102 & 1.2 & 0.8 & 1893 & 0.5 & 0.7 & 1900 & 2.1 & 0.4 \\
\hline
\end{tabular}

${ }^{\mathrm{a}}$ Symbols and terms as explained in Table 3. Tilde indicates samples where the LT torque curve could not be measured.

can be modeled following the function of a weak ferromagnetic moment constrained within the crystal's basal plane (Figure 7f). The ratio of magnetization at room temperature and $77 \mathrm{~K}$ is approximately 1.1 .

\section{Discussion}

[32] Despite the large variation of the hysteresis parameters in the basal plane, $H_{\mathrm{c}}, H_{\mathrm{cr}}, M_{\mathrm{s}}$, and $M_{\mathrm{r}}$, their hysteresis ratios are relatively well confined with high magnetization and low coercivity ratios, similar to those reported in Martin-Hernandez and Guerrero-Suárez [2012] and Ozdemir and Dunlop [2006]. Table 2 summarizes the results from the set of samples analyzed in this study, which indicates that these crystals display a range of all possible crystalline anisotropy structures. $M_{\mathrm{r}} / M_{\mathrm{s}}$ within the basal plane above 0.75 are indicative of a triaxial crystalline anisotropy structure, whereas values close to 0.5 are indicative of a uniaxial crystalline anisotropy structure [Dunlop and Ozdemir, 1997].

[33] Figure 3 shows representative rock magnetic properties for the hematite crystals. In particular, hysteresis loops for the crystals show a reversible behavior at fields higher than $500 \mathrm{mT}$, even for sample HM3044b, the sample with highest coercivity (Table 2). FORC diagrams, however, reveal an asymmetric (kidney) shape and shift in FORC distribution to the field of negative interaction characteristic of unsaturated diagrams [Beron et al., 2011]. The magnetization in the basal plane requires a higher field than suggested from the hysteresis loops, in order to achieve full saturation [Beron et al., 2011].

[34] Table 3 summarizes the initial orientation and magnitude of the WFM at room temperature. Although the reported values appear to be well constrained, with a mean value of $2500 \mathrm{~A} / \mathrm{m}$ [Lin, 1959b; Néel, 1953] the moment from the samples in this work shows a wide range of values. This variation can be explained by several mechanisms that will reduce the parasitic ferromagnetism of hematite, including doping of the lattice with cations other than Fe [Flanders and Remeika, 1965; Tasaki et al., 1962] or oxidation [Tasaki et al., 1960]. A more detailed compositional analysis would be required in order to explore the origin of this variability in the ferromagnetic moment, but it is not allowed for our collection of crystals.

[35] The torque measurements shown in Figure 6 confirm the presence of a WFM constrained within the crystals' basal plane both at room temperature and at $77 \mathrm{~K}$ in five of the eight samples studied. Low-temperature torque curves were previously reported in studies of the Morin transition, magnetic anisotropy and general magnetic properties [Bogdanov, 1973; Flanders and Remeika, 1965; Kaczer and Shalnikova, 1964; Tasaki and Iida, 1963a; Vlasov and Fedoseyeva, 1968]. All of these measurements were concentrated on synthetic crystals with no evidence of a WFM at low temperatures. The origin of the ferromagnetic moment at room temperature has been traditionally explained by the presence of an interaction term described in the Dzyaloshinsky-Moriya model [Dzyaloshinsky, 1958; Moriya, 1960]. However, theory predicts pure antiferromagnetic behavior below the transition temperature. The presence of a moment at low temperature has already been reported in natural crystals [Gallon, 1968; Lin, 1959b; Ozdemir and Dunlop, 2006] but not in synthetic samples [Lin, 1961]. The magnetic moment measured at $77 \mathrm{~K}$ decreases significantly with respect to the values at room temperature (Table 3), but the amount of this decrease varies from one sample to another.

[36] The initial orientation of the ferromagnetic moment is very close to the direction of the first applied field in all the samples with a maximum 
(a) $T>T_{m}$


(b) $T<T_{w}$
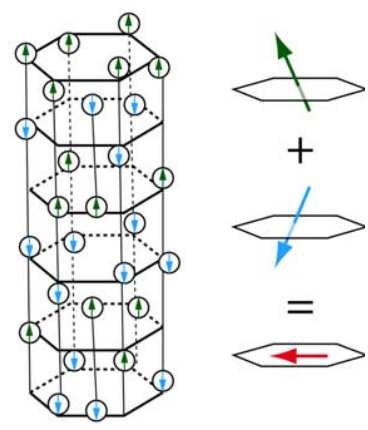

Figure 8. Schematic depiction of the possible spin configuration within the hematite lattice and resulting magnetic moment within alternating lattices (a) above the Morin transition and (b) below the Morin transition. Location of $\mathrm{Fe}^{3+}$ within the hematite lattice modified after Nagata [1961].

deviation of $17.9^{\circ}$ in sample GB4. This initial orientation of the magnetic moment is maintained after rotating the sample into the second measuring position. It is noteworthy that the initial orientation of the magnetization is also close to the direction of the first applied field at low temperatures (Tables 3 and 4). This phenomenon was also reported for low-field susceptibility [Schmidt and Fuller, 1970; Tasaki et al., 1962].

[37] The origin of the unexpected magnetization at low temperatures has long been discussed, and two explanations have been postulated to explain its presence. The first hypothesis is that it is related to defect density, based on the effect that annealing has on the reduction of this magnetization [Néel, 1953; Tasaki et al., 1960]. Because crystallographic twinning has been reported to tilt Bloch walls out of the basal plane of hematite crystals [Tanner et al., 1988], this may be another type of crystalline defect, which is important in preserving a WFM at low temperature. The second idea is that the magnetization has a crystallographic origin [Hirai et al., 1971; Ozdemir and Dunlop, 2006].

[38] The samples in this study show that annealing does reduce the magnetization being particularly important in sample EH02A. This result implies that defects, dislocations, impurities, stress field, and/or twinning influence the WFM measured by torque magnetometry [Dunlop, 1971]. The defect moment in the hematite magnetization therefore has a strong influence in the WFM measured. However, the measured WFM-LT survives annealing, which suggests that there is either another mechanism that also influences the magnetization at low temperatures within the basal plane, or there is no complete removal of the defects by annealing.
[39] Since the WFM is constrained within the basal plane, a more in-depth analysis of the anisotropy within the symmetry plane is needed at the conditions proposed in this work, RT-LT before and after annealing.

[40] Because current theories about the magnetic anisotropy of hematite within the basal plane suggest it is influenced by the magnetoelastic properties [Martin-Hernandez and Guerrero-Suárez, 2012; $\ddot{O} z$ demir, 2008], further work is needed to understand whether the anisotropy within the basal plane controls the WFM at low temperature (Figure 8).

[41] The analysis of the spin orientation at low temperature could be completed by correlating macroscopic torque measurements with neutron diffraction or Mössbauer spectroscopy and determining the microstructural changes and domain structure above and below the transition temperature. Also SEM, microscopy work, or X-ray topography within the basal plane could add in the interpretation of the role played by crystallographic structures in the presence of a WFM-LT.

\section{Conclusions}

[42] The presence of a weak ferromagnetic moment in hematite natural crystals has been detected at $77 \mathrm{~K}$, well below the Morin transition. Classical theories of spin-canted antiferromagnetism predict that hematite should behave like a pure antiferromagnet below the Morin transition. However, the natural hematite crystals in this study show evidence for a WFM moment constrained within the hematite basal plane at $77 \mathrm{~K}$. This WFM moment at low temperature persists even after annealing the samples.

[43] The initial orientation of the WFM moment is parallel, within measurement error, to the orientation of the first applied field. Annealing the samples at $650^{\circ} \mathrm{C}$ reduces the total magnetization from room temperature to $77 \mathrm{~K}$, but a weak WFM persists and maintains the direction of the first applied field. These results suggest that hematite could possibly play a role in planetary magnetism at temperatures below the Morin transition.

\section{Acknowledgments}

[44] The manuscript has benefit from accurate revision by $\ddot{O}$. Özdemir and two anonymous reviewers as well as Associate Editor J. M. Feinberg. S. Guerrero-Suárez is acknowledged 
for assisting rock magnetic measurements. The Geological Museum from Utrecht University and the IGME (Instituto Geológico y Minero de España) are sincerely thanked for lending us some of their samples. This work has been financially supported by a Ramón y Cajal Contract from the Spanish Minister of Science to FMH and SNF project 115897 to AMH.

\section{References}

Bergmüller, F., C. Bärlocher, B. Geyer, M. Grieder, F. Heller, and P. Zweifel (1994), A torque magnetometer for measurements of the high-field anisotropy of rocks and crystals, Meas. Sci. Technol., 5, 1466-1470.

Beron, F., K. R. Pirota, and M. Knobel (2011), Probing the interdependence between irreversible magnetization reversal processes by first-order reversal curves, J. Appl. Phys., 109(7), 109-111.

Besser, P. J., A. H. Morrish, and C. W. Searle (1967), Magnetocrystalline anisotropy of pure and doped hematite, Phys. Rev., 153, 632-640.

Bhathal, R. S. (1971), Magnetic anisotropy in rocks, Earth Sci. Rev., 7, 227-253.

Bilardello, D., and K. P. Kodama (2010a), A new inclination shallowing correction of the Mauch Chunk Formation of Pennsylvania, based on high-field AIR results: Implications for the Carboniferous North American APW path and Pangea reconstructions, Earth Planet. Sci. Lett., 299(1-2), 218227.

Bilardello, D., and K. P. Kodama (2010b), Rock magnetic evidence for inclination shallowing in the early Carboniferous Deer Lake Group red beds of western Newfoundland, Geophys. J. Int., 181(1), 275-289.

Bogdanov, A. A. (1973), Magnetic anisotropy in basal plane hematite, Soviet Phys. Solid State, 14(11), 2845-2848.

Brownlee, S. J., J. M. Feinberg, T. Kasama, R. J. Harrison, G. R. Scott, and P. R. Renne (2011), Magnetic properties of ilmenite-hematite single crystals from the Ecstall pluton near Prince Rupert, British Columbia, Geochem. Geophys. Geosyst., 12, Q07Z29, doi:10.1029/2011GC003622.

Butler, R. (1992), Paleomagnetism, 319 pp., Blackwell Sci., Cambridge.

Coey, J. M. D. (2010), Magnetism and Magnetic Materials, 614 pp., Cambridge Univ. Press, Cambridge.

Collinson, D. W. (1983), Methods in Rock Magnetism and Palaeomagnetism: Techniques and Instrumentation, 503 pp., Chapman and Hall, New York.

Curry, N. A., G. B. Johnston, P. J. Besser, and A. H. Morrish (1965), Neutron diffraction measurements on pure and doped synthetic hematite crystals, Philos. Mag., 12(116), 221-228.

Dunlop, D. J. (1971), Magnetic properties of fine-particle hematite, Ann. Géophys., 27, 269-293.

Dunlop, D. J., and Ö. Özdemir (1997), Rock Magnetism: Fundamentals and Frontiers, 573 pp., Cambridge Univ. Press, Cambridge.

Dzyaloshinsky, I. (1958), A thermodynamic theory of "weak" ferromagnetism of antiferromagnetics, J. Phys. Chem. Sol$i d s, 4,241-255$.

Fabian, K., P. Robinson, S. A. McEnroe, F. Heidelbach, and A. M. Hirt (2011), Experimental study of the magnetic signature of basal-plane anisotropy in hematite, in The Earth's Magnetic Interior, edited by E. Petrovsky et al., pp. 1-17, Springer, New York.
Flanders, P. J. (1972), Observacion of a c-axis moment in alpha-Fe2O3, J. Appl. Phys., 43(5), 2430-2435.

Flanders, P. J., and J. P. Remeika (1965), Magnetic properties of hematite single crystals, Philos. Mag., 11, 1271-1288.

Flanders, P. J., and W. J. Scheuele (1964), Temperature-dependent magnetic properties of hematite single crystals, paper presented at the International Conference on Magnetism, Nottingham, U. K.

Gallon, T. E. (1968), The remanent magnetization of haematite single crystals, Proc. R. Soc. A, 303(1475), 511-524.

Garces, M., J. M. Pares, and L. Cabrera (1996), Further evidence for inclination shallowing in red beds, Geophys. Res. Lett., 23(16), 2065-2068.

Giocavazzo, C. (2011), Fundamentals of Crystallography, 3rd ed., 864 pp., Oxford Univ. Press, Oxford.

Hargraves, R. B. (1959), Magnetic anisotropy and remanent magnetism in hemo-ilmenite from ore deposits at Allard Lake, Quebec, J. Geophys. Res., 64, 1565-1578.

Hirai, A., J. A. Eaton, and C. W. Searle (1971), Fe57 nuclear magnetic resonance and some dynamical characteristics of domain walls in $\alpha-\mathrm{Fe} 2 \mathrm{O} 3$, Phys. Rev. B, 3(1), 68-75.

Jackson, M. J., S. K. Banerjee, J. A. Marvin, R. Lu, and W. Gruber (1991), Detrital remanence, inclination errors, and anhysteretic remanence anisotropy: Quantitative model and experimental results, Geophys. J. Int., 104, 95-103.

Kaczer, J., and T. Shalnikova (1964), Metamagnetism of hematite, Phys. Status Solidi, 6(K177), 589-593.

Kent, D. V., and L. Tauxe (2005), Corrected Late Triassic latitudes for continents adjacent to the North Atlantic, Science, 307(5707), 240-244.

Kletetschka, G., P. J. Wasilewski, and P. T. Taylor (2000), Mineralogy of the sources for magnetic anomalies on Mars, Meteorit. Planet. Sci., 35(5), 895-899.

Kletetschka, G., N. F. Ness, P. J. Wasilewski, J. E. P. Connerney, and M. H. Acuna (2003), Possible mineral sources of magnetic anomalies on Mars, Lead. Edge, 22(8), 766-768.

Kodama, K. P., and W.-W. Sun (1992), Magnetic anisotropy as a correction for compaction-caused paleomagnetic inclination shallowing, Geophys. J. Int., 111, 465-469.

Krása, D., Petersen, K., and N. Petersen (2007), Variable field translation balance, in Encyclopedia of Geomagnetism and Paleomagnetism, Series: Encyclopedia of Earth Sciences Series, Gubbins, D., Herrero-Bervera, E. (Eds.), Springer, pp. 977-979.

Landau, L. (1936), The theory of phase transitions, Nature, $138,840-841$.

Lin, S. T. (1959a), Magnetic properties of hematite single crystals: 1. Magnetization isotherms, antiferromagnetic susceptibility, and weak ferromagnetism of a natural crystal, Phys. Rev., 116(6), 1447-1452.

Lin, S. T. (1959b), Some new magnetic phenomena of hematite single crystal, J. Appl. Phys., 30(4), 306S-307S.

Lin, S. T. (1961), Remanent magnetization of a syntheric hematite single crystal, J. Appl. Phys., 32(3), 394S-395S.

Liu, J., M. I. Richardson, and R. J. Wilson (2003), An assessment of the global, seasonal, and interannual spacecraft record of Martian climate in the thermal infrared, J. Geophys. Res., 108(E8), 5089, doi:10.1029/2002JE001921.

Martin-Hernandez, F., and A. M. Hirt (2001), Separation of ferrimagnetic and paramagnetic anisotropies using a highfield torsion magnetometer, Tectonophysics, 337, 209-221.

Martin-Hernandez, F., and A. M. Hirt (2004), A method for the separation of paramagnetic, ferrimagnetic and hematite magnetic subfabrics using high-field torque magnetometer, Geophys. J. Int., 157(1), 117-127. 
Martin-Hernandez, F., and S. Guerrero-Suárez (2012), Magnetic anisotropy of hematite natural crystals: High field experiments, Int. J. Earth Sci., 10.1007/s00531-00011-00665-z.

Moore, J. M. (2004), Mars-Blueberry fields for ever, Nature, 428(6984), 711-712.

Morin, J. (1950), Magnetic susceptibility of $\alpha-\mathrm{Fe} 2 \mathrm{O} 3$ and Fe2O3 with added titanium, Phys. Rev., 78, 819-820.

Moriya, T. (1960), Anisotropic superexchange interaction and weak ferromagnetism, Phys. Rev., 120, 91-98.

Morrish, A. H. (1994), Canted Antiferromagnetism: Hematite, 192 pp., World Sci., Singapore.

Néel, L. (1953), Some new results on antiferromagnetism and ferromagnetism, Rev. Mod. Phys., 25(1), 58-64.

Owens, W. H. (1981), A simple model for non-vanishing rotational hysteresis in haematite, Phys. Earth Planet. Inter., 27, 106-113.

Owens, W. H., and D. Bamford (1976), Magnetic, seismic and other anisotropic properties of rock fabric, Philos. Trans. $R$. Soc. A, 283, 55-68.

Özdemir, Ö. (2008), Hysteresis and coercivity of multidomain hematite, EOS Trans. AGU, 89(53),, Fall Meeting Suppl. GP31A-0786.

Ozdemir, O., and D. J. Dunlop (2005), Thermoremanent magnetization of multidomain hematite, J. Geophys. Res., 110, B09104, doi : 10.1029/2005JB003820.

Ozdemir, O., and A. Dunlop (2006), Magnetic memory and coupling between spin-canted and defect magnetism in hematite, J. Geophys. Res., 111, B12S03, doi:10.1029/ 2006JB004555.

Peters, C., and M. J. Dekkers (2003), Selected room temperature magnetic parameters as a function of mineralogy, concentration and grain size, Phys. Chem. Earth, 28(16-19), 659-667.

Porath, H., and F. H. Chamalaun (1966), The magnetic anisotropy of hematite bearing rocks, Pure Appl. Geophys., 67, 81-88.

Robinson, P., R. J. Harrison, S. A. McEnroe, and R. B. Hargraves (2002), Lamellar magnetism in the haematiteilmenite series as an explanation for strong remanent magnetization, Nature, 418, 517-520.

Rochette, P., P. E. Mathe, L. Esteban, H. Rakoto, J. L. Bouchez, Q. Liu, and J. Torrent (2005), Non-saturation of the defect moment of goehtite and fine-grained hematite up to
57 teslas, Geophys. Res. Lett., 32, L2239, doi:10.1029/ 2005GL024196.

Schmidt, and M. D. Fuller (1970), Low-field susceptibility anisotropy in the basal plane of hematite (a-Fe2O3) and its dependence on the remanent moment, J. Appl. Phys., 41(3), 994-995.

Schmidt, V., A. M. Hirt, K. Hametner, and D. Gunther (2007a), Magnetic anisotropy of carbonate minerals at room temperature and 77 K, Am. Mineral., 92(10), 1673-1684.

Schmidt, V., A. M. Hirt, P. Rosselli, and F. Martin-Hernandez (2007b), Separation of diamagnetic and paramagnetic anisotropy by high-field, low-temperature torque measurements, Geophys. J. Int., 168(1), 40-47.

Squyres, S. W., et al. (2009), Exploration of Victoria Crater by the Mars Rover Opportunity, Science, 324(5930), 10581061.

Stacey, F. D., and S. K. Banerjee (1974), The Physical Principles of Rock Magnetism, 195 pp., Elsevier, Amsterdam.

Tanner, B. K., G. F. Clark, and M. Safa (1988), Domain structures in haematite (a-Fe2O3), Philos. Mag. B, 57(3), 361377.

Tasaki, A., and S. Iida (1963a), Magnetic properties of synthetic single crystal of $\alpha \mathrm{Fe} 2 \mathrm{O} 3$, J. Phys. Soc. Jpn., 18(8), $1148-1154$.

Tasaki, A., and S. Iida (1963b), Magnetic properties of synthetic single crystal of alpha Fe2O3, J. Phys. Soc. Jpn., 18(8), 1148-1154.

Tasaki, A., K. Siratori, and S. Iida (1960), The influence of oxidation and reduction on the parasitic ferromagnetism of $\alpha-\mathrm{Fe} 2 \mathrm{O} 3$, J. Phys. Soc. Jpn., 15, 1535.

Tasaki, A., K. Siratori, and S. Iida (1962), Experimental study of parasitic ferromagnetism of $\alpha-\mathrm{Fe} 2 \mathrm{O} 3$, J. Phys. Soc. Jpn., 17(Suppl. B-I), 235-238.

Tauxe, L., K. P. Kodama, and D. V. Kent (2008), Testing corrections for paleomagnetic inclination error in sedimentary rocks: A comparative approach, Phys. Earth Planet. Inter., 169(1-4), 152-165.

Townsend, T. (1920), Magnetization and hysteresis in hematite crystals, Phys. Rev., 15(5), 345-364.

Vlasov, A. Y., and N. V. Fedoseyeva (1968), The temperature dependence of the constant of crystallographic magnetic anisotropy of hematite, Izv. Earth Phys., 5, 108-111. 